\title{
ANÁLISE DO ACIONAMENTO ELÉTRICO EMPREGADO EM SISTEMAS DE AGITAÇÃO MECÂNICA
}

\author{
A. B. LA ROSA ${ }^{1}$, G. DORS ${ }^{1}$ e M. ESPOSITO ${ }^{1}$ \\ ${ }^{1}$ Universidade Federal de Pelotas, Centro de Engenharias \\ E-mail para contato: marcelo.esposito@ufpel.edu.br
}

\begin{abstract}
RESUMO - Diferentes tipos de mecanismos de acionamento podem ser empregados em operações de agitação ou mistura, sendo os motores elétricos com transmissão direta ou acoplados a redutores mecânicos de velocidade os mais utilizados. Outro dispositivo que pode ou não fazer parte do circuito elétrico são os inversores de frequência. Além da potência consumida pelo fluido, outras parcelas devem ser contabilizadas na determinação da potência instalada requerida pelo sistema: a potência necessária para vencer a inércia mecânica e a potência consumida por atritos em mancais, selos, gaxetas e engrenagens. A unidade experimental é formada por motores de indução de 2 a 8 polos de $0,25 \mathrm{~kW}$ e um analisador de energia. $\mathrm{O}$ comportamento dinâmico das grandezas elétricas durante o funcionamento em regime permanente e/ou frenagens do eixo dos motores elétricos trifásicos de corrente alternada serão apresentados. Os dados experimentais demonstram claramente que a corrente que percorre o circuito elétrico do sistema de agitação pode ser utilizada para identificar anomalias indesejadas durante sua operação.
\end{abstract}

\section{INTRODUÇÃO}

A agitação está presente em diversas áreas das indústrias, seja como mais uma etapa do processo, ou como a parte crítica na obtenção do produto final com as especificações desejadas. A agitação em si é um processo que fornece movimento ao líquido sendo responsável pela realização dos processos químicos e físicos em diversas aplicações. Cinco aplicações da agitação são apresentadas na Tabela 1:

Tabela 1 - Aplicações e processamentos relacionados ao sistema de agitação

\begin{tabular}{|l|c|c|}
\hline Aplicação & Processamento físico & Processamento químico \\
\hline Líquido-sólido & Suspensão & Dissolução \\
\hline Líquido-gás & Dispersão & Absorção \\
\hline Líquidos imiscíveis & Emulsão & Extração \\
\hline Líquidos miscíveis & Mistura & Reação \\
\hline Movimentação do fluido & Bombeamento & Transferência de calor \\
\hline
\end{tabular}

Fonte: Joaquim Júnior et al., 2007

Um tanque agitado convencional consiste regularmente em um tanque cilíndrico, tampos, um ou mais impelidores, um motor e em ocasiões específicas chicanas. Alguns tanques são equipados de serpentinas ou camisas para troca térmica. Além da geometria, outras variáveis que são importantes no projeto de um tanque agitado são as propriedades do fluido: viscosidade, 
densidade, tensão superficial, condutividade térmica e calor específico. Além destas deve-se considerar também a velocidade de rotação do impelidor, potência, torque, velocidade relativa entre a pá do impelidor e o fluido e força de arraste do impelidor.

Uma característica muito importante no dimensionamento do sistema de agitação é a forma de carregamento que pode ser: instantânea, fracionada ou contínua. Um exemplo de um possível problema a ser gerado durante o carregamento está relacionado com a adição de elementos sólidos, no caso do carregamento instantâneo. Isso pode ocasionar a sobrecarga do impelidor, exigir maior torque e, portanto maior resistência mecânica. Outra situação é a partida em meio de sólidos decantados. Em um processo de suspensão de sólidos geralmente o material sólido é adicionado à mistura aos poucos para facilitar a suspensão, caso ocorra à parada da agitação devido à falha mecânica ou elétrica terá início a decantação dos sólidos, até então suspensos no meio líquido. Quando o sistema for posto em funcionamento novamente, com os sólidos depositados no fundo do tanque, o agitador estará imerso em meio sólido, este evento pode vir a impedir o funcionamento ou até mesmo comprometer a estrutura mecânica do sistema. Uma abordagem de grande importância é relacionar a corrente elétrica com a resistência ao giro do eixo do motor, esta informação disponibilizada em tempo real pode evitar situações indesejadas como as citadas (Joaquim Júnior et al., 2007).

\subsection{Reações de Polimerização: Identificação da Coalescência do Meio Reacional}

No caso das reações de polimerização a falta de estabilidade do látex pode levar a coalescência total ou parcial do meio reacional. Dependendo da forma, do tamanho e da quantidade de coágulos e/ou camadas de polímero que vierem a se formar dentro do reator, o prejuízo pode ser muito superior a produção de um produto de baixa qualidade. No trabalho de Esposito (2010) a forte coalescência fez com que o PT100 utilizado para a medição da temperatura do reator fosse danificado e sua haste carregada pelo sistema de agitação. Somente com a medição da corrente elétrica alternada que percorre o circuito do sistema de agitação, corrente de entrada do inversor de frequência que está ligado a uma rede monofásica, foi possível perceber que alguma anomalia estava ocorrendo dentro do reator.

A corrente elétrica que percorre o circuito do sistema de agitação é comumente utilizada para inferir a viscosidade de um meio. Bhat et al. (2006a, b, c) utilizaram dados de temperatura, de uma polimerização em massa via radicais livres de MMA (Metacrilato de metila) em batelada, e medidas da corrente elétrica para desenvolver um soft sensor capaz de efetuar a estimativa em linha da conversão e do peso molecular médio em condições isotérmicas e não-isotérmicas. Cabe ressaltar que nos trabalhos citados a técnica de medição da corrente elétrica não foi descrita em detalhe, mas pela forma como a potência foi calculada, subentende-se que a agitação foi efetuada utilizando-se um motor de corrente contínua (cc).

A maioria dos reatores de grande porte, senão todos, são equipados com motores de corrente alternada (ca) e neste caso a medição da potência não é tão trivial. Isso porque nos cálculos deve constar um termo adicional chamado de fator de potência, que é definido como a razão entre a potência ativa e a potência aparente. Geralmente, o valor do fator de potência a plena carga (100\%) é mostrado na placa de identificação do motor (valores nominais), entretanto, a principal dificuldade é a atualização deste fator de acordo com a variação da carga aplicada ao eixo do 
motor (Ascanio et al., 2004, Kowalski, 2009).

No presente trabalho, os equipamentos elétricos que fazem parte do sistema de agitação mecânica são: um inversor de frequência e um motor de indução trifásico de corrente alternada. De posse dos dados de desempenho do motor, fornecidos pelo fabricante, constata-se que há um aumento expressivo da corrente elétrica em função do aumento da carga aplicada ao eixo do motor. No entanto, esta relação depende do tipo de carga e se o acoplamento é direto ou com redutor. Aplicações onde motores autoventilados devem desenvolver pleno torque com rotação abaixo da nominal, podem causar sobreaquecimento, já que o fluxo de ar disponível para refrigeração também fica reduzido. O inversor foi utilizado para dar maior versatilidade, com relação à possibilidade de variação da velocidade e também como proteção contra sobrecarga do motor.

Devido às características do circuito de entrada da maioria dos inversores, que é constituída por um retificador a diodos e um banco de capacitores, a sua corrente de entrada possui uma forma de onda não senoidal. O conjunto motor mais inversor de frequência é visto pela fonte de alimentação como uma carga não linear, cuja corrente possui harmônicas (componentes de frequências múltiplas da frequência da rede). Do ponto de vista do motor tem-se uma tensão pulsada e uma corrente praticamente senoidal, portanto as harmônicas de maior amplitude no motor são de tensão. Neste contexto, a instalação e utilização de inversores de frequência exigem certos cuidados de forma a se evitar a ocorrência de interferência eletromagnética (devido ao conteúdo harmônico), que é caracterizada pelo distúrbio no funcionamento normal dos inversores ou de outros consumidores (WEG, 2006 e 2009).

A medição da corrente elétrica alternada em circuitos que possuem cargas não lineares deve ser vista com atenção, uma vez que os transdutores convencionais utilizam o valor rms, isto é, com o valor de pico da forma de onda multiplicado por 0,707 , reproduzido na saída o valor eficaz. Este método é eficiente exclusivamente para forma de onda senoidal. Em situações com formas de ondas distorcidas, os transdutores com medição rms podem apresentar erros de medição de mais de 50\%. Para estas situações, é recomendado o uso de transdutores com medição true rms (KRON, 2007).

Ao se fazer uso de analisadores de energia pretende-se: detectar e evitar problemas de qualidade de energia antes que causem paradas na operação, validar a qualidade de energia no ponto de entrada da rede elétrica, detectar distúrbios intermitentes e difíceis de localizar e verificar a capacidade do sistema elétrico antes da aplicação de cargas. Além de medir a potência reativa (causada pelo baixo fator de potência), também é possível medir o desperdício de energia causado pelo desequilíbrio e o efeito do carregamento desigual de cada fase em sistemas trifásicos. Efetuar a aquisição de sinais de tensão, corrente e frequência simultaneamente o que possibilita que a dinâmica do sistema seja caracterizada. Distúrbios de energia podem envolver tensão, corrente ou frequência e, normalmente, se manifestam como quedas, ondulações, distorção harmônica, desequilíbrio, tremulações e transientes.

A medição de grandezas elétricas para inferir mudanças no meio reacional é especialmente interessante porque as medidas não afetam a reação, apresentam um baixo custo para sua implementação, são rápidas e contínuas e podem ser facilmente interpretadas. O objetivo do presente trabalho é identificar quais grandezas elétricas devem ser medidas, como interpretar a informação contida neste sinal e principalmente determinar o local, no circuito elétrico, onde os 
instrumentos de medição devem ser instalados em sistemas com agitação mecânica.

\section{MATERIAL E MÉTODOS}

Para a validação dos resultados foi utilizado um analisador de qualidade de energia e potência trifásico, marca Fluke, modelo 434 série II. Foram utilizadas as seguintes funções de medição: (1) Volt/Amps/Hertz; (2) Quedas e Picos - Dips \& Swells; (3) Harmônicos; (4) Potência e Energia; (5) Calculadora de Perda de Energia; (6) Desequilíbrio; (7) Irrupção - Inrush; (8) Monitor. Para a transferência de dados registrados fez-se uso do software POWER LOG que basicamente apresenta as seguintes telas para cada uma das oito funções utilizadas: tela principal de qualidade de potência, tela de medidor, tela de gráfico de barras, tela do fasor, tela de tendência e tela de eventos. Os equipamentos utilizados nos ensaios foram: um inversor de frequência marca/modelo VACON/0010-1L-0005-2-MACHINERY, 220Vca, 1,5CV/1,1kW, 4,8Aca e motores de indução trifásicos da marca VOGES, 1/3 CV, 220/380Vca, $60 \mathrm{~Hz}$ de 2, 4, 6 e 8 polos, ou seja, rotação nominal de 3360, 1680, 1110 e 860 rpm, respectivamente, e um motofreio trifásico modelo MFB 63 A2, 2 polos, 1/3 CV, com freio tipo MFC 63, 220 Vca, 0,08 Aca, monofásico. Utilizando o conjunto de equipamentos mostrado na Figura 1 foram realizadas 29 ligações (ensaios) totalizando 232 experimentos.

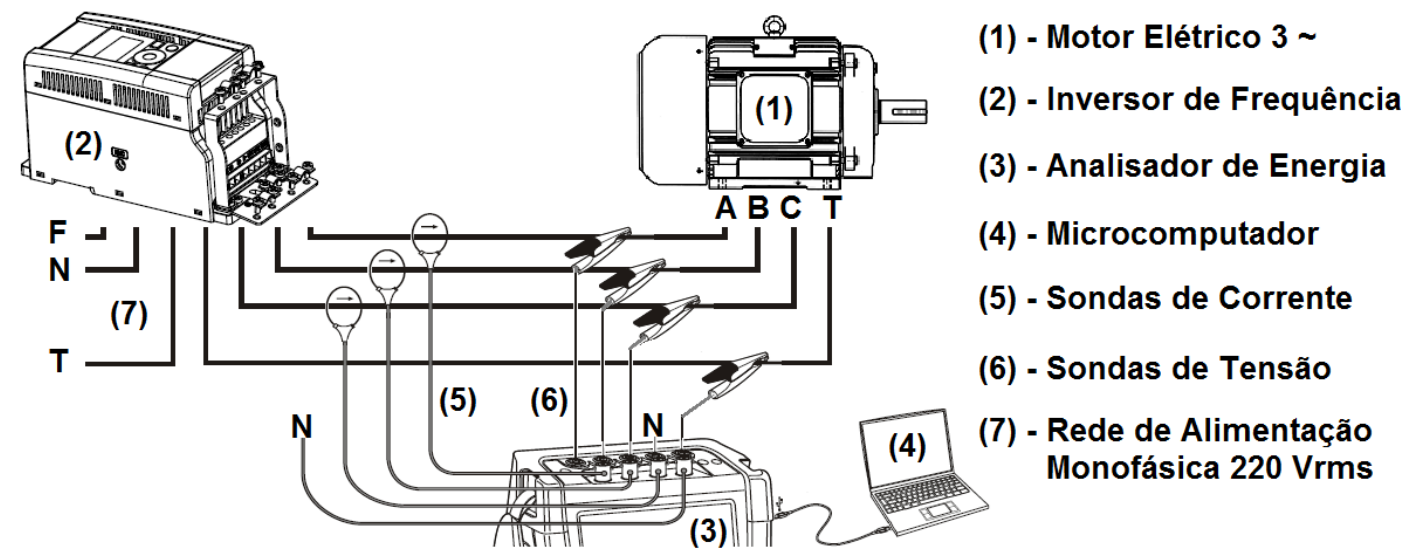

Figura 1 - Ligação do analisador de energia em triângulo, sem neutro.

\section{RESULTADOS E DISCUSSÃO}

A Tabela 2 apresenta as 29 ligações efetuadas. Cada ensaio representa um modo de operação do sistema de agitação mecânica. Destacam-se a utilização de motores elétricos de mesma potência, porém com diferentes velocidades nominais e a ligação em diferentes pontos do circuito elétrico dos sensores de medição de grandezas elétricas. Esta análise serviu principalmente para se verificar a propagação do ruído nas medições, a repetibilidade dos resultados, a estabilidade e principalmente a sensibilidade da medição da corrente elétrica com a rotação ou a força aplicada ao eixo do motor.

Também fez parte deste trabalho a identificação de grandezas elétricas que possibilitem a obtenção plena dos objetivos propostos, desta forma dentre as várias funções de medição do analisador de qualidade de energia a mais utilizada foi a (3) Harmônicos, mais especificamente, os gráficos de tensão e corrente e histogramas de harmônicas. Harmônicas são distorções periódicas 
de ondas senoidais de tensão, corrente ou potência. Uma forma de onda pode ser considerada uma combinação de várias ondas senoidais de diferentes frequências e magnitudes. Um sinal sem distorção deve mostrar a $1^{\text {a }}$ harmônica (a fundamental) com 100\%, e as outras com zero. Uma onda senoidal pura é distorcida quando componentes de frequência mais alta são adicionadas a ela.

Tabela 2 - Análise do acionamento elétrico empregado em sistemas de agitação mecânica

\begin{tabular}{|c|c|c|c|c|c|c|c|c|c|c|c|c|c|c|c|}
\hline \multicolumn{6}{|c|}{$\begin{array}{l}\text { Número de polos } \\
\text { Motor elétrico de indução } \\
\text { trifásico. }\end{array}$} & \multicolumn{3}{|c|}{$\begin{array}{l}\text { Ligação } \\
\text { Analisador } \\
\text { de energia. }\end{array}$} & \multirow{2}{*}{$\begin{array}{l}\text { Inversor } \\
\text { de } \\
\text { frequência }\end{array}$} & \multicolumn{6}{|c|}{$\begin{array}{l}\text { Rotação - ou + = sentido horário ou anti-horário. } \\
\text { No sentido - ou + a frenagem ocorreu em 45Hz. }\end{array}$} \\
\hline & $\begin{array}{l}\text { reio } \\
\text { los }\end{array}$ & 2 & 4 & 6 & 8 & $\Delta$ & $\mathrm{Y}$ & $1 \sim$ & & Frenagem & Reversão & $60 \mathrm{~Hz}$ & $30 \mathrm{~Hz}$ & $\begin{array}{l}-60 \mathrm{~Hz} \\
\text { à } \\
30 \mathrm{~Hz}\end{array}$ & $\begin{array}{l}+60 \mathrm{~Hz} \\
\text { à } \\
30 \mathrm{~Hz}\end{array}$ \\
\hline 01 & $X$ & & & & & $\mathrm{X}$ & & & $X$ & $X$ & & X & & & \\
\hline 02 & $\mathrm{X}$ & & & & & $X$ & & & $X$ & & $X$ & $X$ & & & \\
\hline 03 & $\mathrm{X}$ & & & & & $\mathrm{X}$ & & & $X$ & $X$ & & & $\mathrm{X}$ & & \\
\hline 04 & $X$ & & & & & $X$ & & & $X$ & & $X$ & & $\mathrm{X}$ & & \\
\hline 05 & $\mathrm{X}$ & & & & & $X$ & & & $X$ & $X$ & & & & & $\mathrm{X}$ \\
\hline 06 & $X$ & & & & & $\mathrm{X}$ & & & $X$ & $\mathrm{X}$ & & & & X & \\
\hline 07 & $X$ & & & & & & $X$ & & & $X$ & & $X$ & & & \\
\hline 08 & $X$ & & & & & & $\mathrm{X}$ & & & & & X & & & \\
\hline 09 & $X$ & & & & & & & $X$ & $X$ & & $X$ & & $X$ & & \\
\hline 10 & $X$ & & & & & & & X & X & $X$ & & & $X$ & & \\
\hline 11 & $X$ & & & & & & & $X$ & $X$ & $X$ & & X & & & \\
\hline 12 & $\mathrm{X}$ & & & & & & & $\mathrm{X}$ & $X$ & $X$ & & & & & $X$ \\
\hline 13 & $X$ & & & & & & & $X$ & $X$ & $X$ & & & & $X$ & \\
\hline 14 & & $X$ & & & & & $X$ & & & & & $X$ & & & \\
\hline 15 & & X & & & & $X$ & & & X & & $X$ & X & & & \\
\hline 16 & & $\mathrm{X}$ & & & & $X$ & & & $X$ & & & & & & $X$ \\
\hline 17 & & X & & & & & & X & X & & & & & & $X$ \\
\hline 18 & & & $X$ & & & & $X$ & & & & & $X$ & & & \\
\hline 19 & & & $X$ & & & $X$ & & & $X$ & & $X$ & X & & & \\
\hline 20 & & & $X$ & & & $X$ & & & $X$ & & & & & & $X$ \\
\hline 21 & & & $X$ & & & & & $X$ & $X$ & & & & & & $X$ \\
\hline 22 & & & & $X$ & & & $X$ & & & & & X & & & \\
\hline 23 & & & & $X$ & & $X$ & & & $X$ & & $X$ & $X$ & & & \\
\hline 24 & & & & $X$ & & $X$ & & & $X$ & & & & & & $X$ \\
\hline 25 & & & & $X$ & & & & $X$ & $X$ & & & & & & $X$ \\
\hline 26 & & & & & $X$ & & $X$ & & & & & X & & & \\
\hline 27 & & & & & $X$ & $X$ & & & $X$ & & $X$ & $X$ & & & \\
\hline 28 & & & & & $X$ & $\mathrm{X}$ & & & $X$ & & & & & & $X$ \\
\hline 29 & & & & & $X$ & & & $X$ & $X$ & & & & & & $X$ \\
\hline
\end{tabular}

No caso da ligação trifásica 380 Vrms em estrela (Y), sem neutro e sem o uso de inversor de frequência, o analisador de energia foi ligado entre a rede de alimentação trifásica e o motor. Como neste caso o motor foi energizado com valores nominais de tensão, o mesmo operou em velocidade de rotação nominal. Comparando-se, por exemplo, o ensaio 26 com o motor de 8 polos, com o ensaio 07 utilizando o motofreio de 2 polos, não foi possível identificar, pelo histograma referente as harmônicas, que se tratam de ensaios diferentes. Na comparação entre os gráficos (Figura 2) referentes às tensões e correntes medidas nestes ensaios, percebe-se nitidamente a frenagem, que ocorreu de forma instantânea, por um período de $3 \mathrm{~s}$, do eixo do motor no ensaio 07 . A diferença é que ao invés de valores com pequena oscilação, observou-se no momento da frenagem uma queda sensível da tensão e uma elevação abrupta da corrente nas três fases, que retorna ao valor nominal após a liberação do eixo. A mesma conclusão pode ser obtida da comparação do ensaio 07 com os ensaios 08, 14, 18 e 22. 

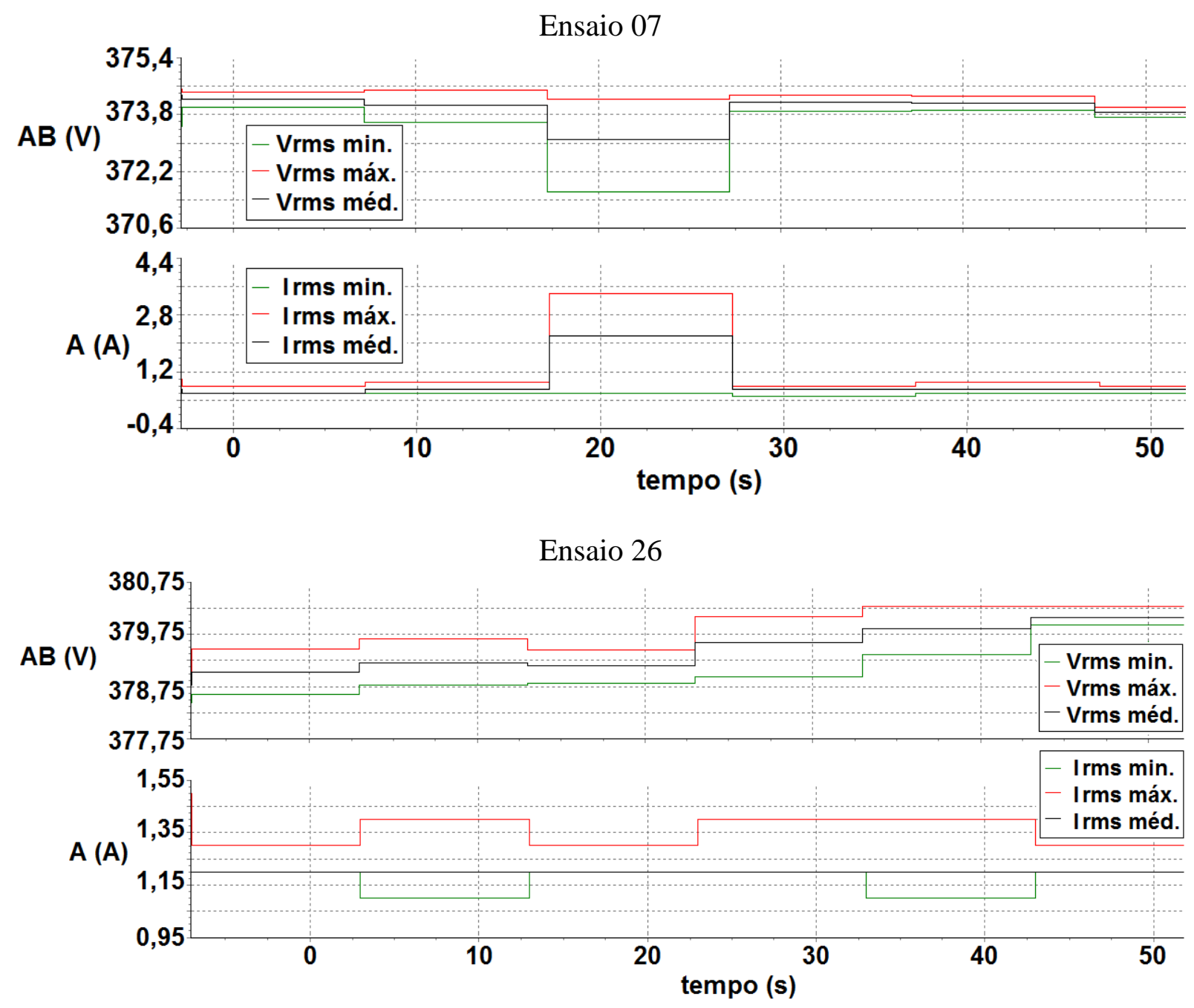

Figura 2 - Comportamento dinâmico da tensão (AB) e da corrente (A) nos ensaios 07 e 26.

A alimentação de entrada do inversor de frequência é monofásica (1 Vin = 208-240Vca) e a saída é trifásica (3 Vout $=0$-Vin $0-320 \mathrm{~Hz}$ ). Deste modo, realizaram-se ensaios com o analisador de energia ligado entre a rede de alimentação monofásica (220 Vrms) e o inversor de frequência. O objetivo destes ensaios foi estudar o comportamento dinâmico da carga não linear e comparar com o caso em que os dados de tensão e corrente são adquiridos entre o inversor e o motor. Em ambos os ensaios, 12 e 13, identificou-se o travamento do eixo (frenagem) pela elevação abrupta da corrente. A variação da velocidade de rotação do eixo do motor (ou da frequência de alimentação), função destinada ao inversor, foi observada pelo histograma das harmônicas. Nos dois ensaios, independentemente do sentido de rotação, a tensão apresentou sua harmônica fundamental correspondendo a aproximadamente $100 \%$ do sinal, ou seja, distorção harmônica total abaixo de 5\%. Enquanto que a corrente apresentou distorção harmônica total próxima de $90 \%$. Os resultados obtidos não permitiram a identificação da redução da velocidade, somente a presença no circuito do inversor de frequência. Resultados semelhantes foram obtidos nos ensaios 10 e 11. Nos ensaios 17, 21, 25 e 29 os valores de tensão e corrente permaneceram praticamente inalterados com relação aos seus valores nominais. Todos os ensaios foram realizados com o motor já em regime de trabalho e em sua velocidade nominal, que foi reduzida até a metade do seu 
valor em um intervalo de tempo de um minuto. Novamente a existência do inversor foi identificada analisando-se a distorção harmônica da corrente. O ensaio 09 forneceu resultados semelhantes a estes em que não houve a frenagem do eixo.

A ligação do analisador de energia entre o motor e o inversor de frequência, no caso da ligação trifásica 220 Vrms em triângulo ( $)$ ), dificultou, mas ão inviabilizou a identificação da frenagem ou da reversão no sentido de rotação ou da redução de velocidade do eixo do motor, devido ao aumento no número de componentes harmônicas dos sinais medidos. A comparação entre os resultados obtidos nos ensaios 02, 15, 19, 23 e 27, revelou que é possível identificar claramente a reversão no sentido de rotação do eixo do motor observando-se o comportamento dinâmico da tensão nas três fases. Durante a rampa de desaceleração e posterior aceleração, aplicadas no procedimento de reversão, a tensão decresce de 220 Vrms para um valor próximo de zero volts. Na ligação triângulo, sem neutro, as tensões de linha e de fase são iguais. Os valores de corrente não divergiram durante os ensaios. Com relação as harmônicas a distribuição em três fases diminuiu a distorção harmônica total da corrente para valores entre 20 e 30\%. É importante destacar que no caso do ensaio 09 a reversão não pode ser identificada analisando-se apenas a evolução temporal das grandezas medidas, pois não houve uma queda de tensão na alimentação do inversor de frequência nem uma mudança considerável no valor rms da corrente. No ensaio 04, com o motor operando na metade da sua velocidade nominal $(30 \mathrm{~Hz})$ a redução da tensão foi de 132 Vrms para 68 Vrms, enquanto que a corrente não apresentou alteração, permanecendo próxima de 1,3 Arms nas três fases. Outro fato que chama a atenção é a possibilidade de identificação da reversão utilizando-se os dados de distorção harmônica de tensão que aumenta de 11 para 36\% e/ou de corrente que aumenta de 18 para 32\% somente durante o procedimento de reversão no sentido de rotação.

Nos ensaios 16, 20, 24 e 28 a ligação do analisador de energia entre o inversor e o motor possibilitou a identificação da redução da velocidade de rotação do eixo do motor, desde o valor nominal até o limite inferior de $30 \mathrm{~Hz}$ (restrição com relação à ventilação do motor). A partir do momento em que a redução gradual da velocidade é iniciada, nota-se uma redução no valor rms da tensão e um aumento no valor rms da corrente, nas três fases. Nos ensaios 01, 03, 05 e 06 além da frenagem que pode ser detectada realizando a medição dos valores true rms da tensão e da corrente no circuito triásico foi possível, nos ensaios 05 e 06, identificar a redução da velocidade de rotação, porém sem determinar o sentido de rotação e o momento exato do travamento do eixo, uma vez que a tensão já está em queda e a corrente não forneceu informações para tanto.

\section{CONCLUSÕES}

Conclui-se que a medição da tensão e da corrente true rms diretamente nos bornes de alimentação de motores de indução, monofásicos ou trifásicos, conectados ou não a inversores de frequência, representa a melhor opção para o objetivo proposto, monitoramento de sistemas com agitação mecânica. De antemão informações sobre o travamento da haste e do impelidor e sobre a variação da velocidade de rotação, podem facilmente ser obtidas e interpretadas. O próximo passo será estender esta análise para motores com redutores mecânicos de velocidade, utilizados industrialmente para garantir o torque em baixas rotações e desenvolver um estudo sobre a aplicação da técnica em meios agitados.

Diante do exposto percebe-se que os resultados e os impactos esperados vão muito além da medição de grandezas elétricas para inferir variações no meio em sistemas agitados. O foco 
principal é evitar o desperdício de reagentes e evitar a parada desnecessária da planta ou processo. Embora exaustivos testes, em unidades experimentais piloto sejam realizados previamente, nem sempre é possível prever se pequenas alterações na receita do produto final vão ou não gerar situações de risco ao operador, no caso da agitação de produtos químicos nocivos ao ser humano, ou se as consequências serão o entupimento de válvulas e motobombas e no pior caso danos ao reator e seus periféricos. A medição da corrente elétrica que percorre o circuito elétrico do sistema de agitação pode fornecer informações suficientes, em tempo real, para evitar situações potencialmente perigosas e onerosas do ponto de vista do custo operacional. No entanto é notável que ao se efetuar medidas elétricas e analisá-las, outras importantes conclusões podem ser extraídas, principalmente por causa da conexão entre os circuitos elétricos. Sugestões e/ou correções podem resultar como consequência destas medições, por exemplo, na redução do custo da energia elétrica consumida pelo sistema de agitação com a correção do fator de potência.

\section{AGRADECIMENTOS}

Os autores agradecem CNPq - Conselho Nacional de Desenvolvimento Científico e Tecnológico pelo apoio financeiro. A empresa VOGES MOTORES pela doação dos motores elétricos e a empresa ALTUS SISTEMAS DE AUTOMAÇÃO S.A. pela doação do inversor de frequência.

\section{REFERÊNCIAS}

ASCANIO, G.; CASTRO, B.; GALINDO, E. Measurement of power consumption in stirred vessels - a review. Chem. Eng. Res. Des. v. 82, p. 1282-1290, 2004.

BHAT, S. A.; SARAF, D. N.; GUPTA, S.; GUPTA, S. K. On-line optimizing control of bulk free radical polymerization reactors under temporary loss of temperature regulation: experimental study on a 1-L batch reactor. Ind. Eng. Chem. Res. v. 45, p. 7530-7539, 2006 a.

BHAT, S. A.; SARAF, D. N.; GUPTA, S.; GUPTA, S. K. Use of agitator power as a soft sensor for bulk free-radical polymerization of methyl methacrylate in batch reactors. Ind. Eng. Chem. Res. v. 45, p. 4243-4255, 2006 b.

BHAT, S. A.; SARAF, D. N.; GUPTA, S.; GUPTA, S. K. Some practical aspects of designing a laboratory scale batch polymerization reactor without gas entrapment and interfaced with virtual instrumentation. ISA T. v. 45, p. 259-269, 2006c.

ESPOSITO, M., Instrumentação e monitoramento em tempo real de reatores de polimerização através de técnicas calorimétricas. 2010. 155f. Tese (Doutorado em Engenharia Química). Universidade Federal de Santa Catarina, Florianópolis.

JOAQUIM JÚNIOR, C. F.; CEKINSKI, E.; NUNHEZ, J. R.; URENHA, L. C. Agitação e mistura na indústria. Editora LTC, 2007.

KRON. Manual do usuário - Transdutor de tensão ou corrente alternada (analógico), rev. 2, 2007.

KOWALSKI, A. J. An expression for the power consumption of in-line rotor-stator devices, Chem. Eng. Process. v. 48, p. 581-585, 2009.

WEG. Manual do usuário - Inversor de frequência CFW-08, Software ver. 4.1X, cód. 0899.5241 $\mathrm{P} / 9,2006$.

WEG. Guia técnico - Motores de indução alimentados por inversores de frequência PWM, cód. 027/00, 2009. 